\title{
Thyroid hormones and the heart
}

\author{
Gheorghe-Andrei Dan ${ }^{1}$
}

Published online: 21 April 2016

(c) Springer Science+Business Media New York 2016

There are more things in heaven and earth, Horatio,

Than are dreamt of in your philosophy.

\section{-Hamlet (1.5.167-8), Hamlet to Horatio}

Thyroid and heart share a common embryologic origin and an intimate and complex functional relationship [1]. Cardiovascular effects and clinical signs are the most prominent features of the thyroid dysfunction. Not only dysregulation of thyroid hormones are risk factors for cardiac disease, but there is a mutual relationship and dysregulation of thyroid hormones is also a marker for chronic heart disease. Indeed, chronic heart disease is accompanied by a decrease in $\mathrm{T}_{3}$ (low $T_{3}$ syndrome) with consequent negative effects on cardiac and vascular functions [2]. This non-thyroid related decrease of $T_{3}$ is attributed to the impairment of the hepatic deiodinase; interestingly, this enzyme is induced through transcriptional genomic actions of $\mathrm{T}_{3}$, but its action is blocked by cytokines in chronic diseases [3]. Thyroid hormone synthesis is regulated by thyroid-stimulating hormone (TSH) through a feedback mechanism; however, this feedback is driven by $\mathrm{T}_{4}$ and therefore in low $T_{3}$ syndrome a compensatory pituitary response could not be elicited. Moreover, increase in $T_{4}$ production has no effect on $T_{3}$ level as the conversion is impaired [2].

There are two main routes for thyroid hormone action: the transcriptional genomic effects and the non-genomic effects, the last targeting directly different cell structures.

Gheorghe-Andrei Dan

andrei.dan@gadan.ro

1 Medicine Faculty, Colentina University Hospital, University of Medicine "Carol Davila", Bucharest, Romania
The thyroid hormone receptors bind to the thyroid hormone response elements in the promoter region of specific genes, activating or repressing gene expression [4]. Only $\mathrm{T}_{3}$ enters the myocyte, via transport proteins (belonging to the surface protein transporters family) [5] and mediates the cellular effects as cardiomyocyte cannot metabolize $T_{4}$ to $T_{3}$. Many essential myocyte genes are regulated by $T_{3}$, and this explains the prompt and important myocardial reaction to thyroid hormones; a list of the most important regulated proteins is presented in Table 1.

The combined action on SERCA and phospholamban favoring calcium reuptake confers to $\mathrm{T}_{3}$ a unique role in regulation of cardiac relaxation. Consequently, it appears that in low $T_{3}$ syndrome accompanying chronic heart disease decreased $T_{3}$ concentration induces diastolic dysfunction and therefore represents a risk factor for heart failure progression. Main inotropic actions of thyroid hormones are also mediated through the modulation of calcium re-uptake and are dissociated from the beta adrenergic actions [6]. As mentioned, $T_{3}$ has also equally important regulatory action on different cell key signaling molecules such as phosphatidylinositol 3 kinase/protein kinase $\mathrm{B}$ (PI3 K/Akt), extracellular signal-regulated kinases (ERK1/2), and p38 mitogen-activated protein kinase (p38MAPK) or tumor suppressor protein p53.

In addition to these local actions thyroid hormones exert significant systemic hemodynamic actions, mainly mediated by $\mathrm{T}_{3}$, acting, thus, on the cardiovascular system as a whole. Through its action on thermogenesis $\mathrm{T}_{3}$ decreases vascular resistance and cardiac afterload, contributing to regulation of cardiac output and inotropy. Secondary RAAS activation increases sodium reabsorption and subsequently blood volume with beneficial effects on cardiac 
Table 1 Cardiac-specific proteins regulated by $\mathrm{T}_{3}$ (after [2])

\begin{tabular}{ll}
\hline Transcriptional activation & Transcriptional repression \\
\hline Alpha myosin heavy chain & Beta myosin heavy chain \\
Sarcoplasmic reticulum $\mathrm{Ca}^{2+}$-ATPase (SERCA) & Phospholamban \\
$\mathrm{Na}^{+} / \mathrm{K}^{+}$ATPase & Adenyl cyclase catalytic subunits \\
Beta1-adrenoreceptor & Thyroid nuclear receptor alpha- \\
Atrial natriuretic peptide & $\mathrm{Na}^{+} / \mathrm{Ca}^{2+}$ exchanger \\
Guanine nucleotide regulatory proteins & Thyroid hormone transporter (MTC family) \\
Voltage gated $\mathrm{K}^{+}$channel & Adenine nucleotide translocase-1 \\
\hline
\end{tabular}

work by maintaining the preload. $\mathrm{T}_{3}$ also exerts hemodynamic mediated positive chronotropic effects. If the local $\mathrm{T}_{3}$ actions (at the cell level) are responsible for the more subtle and long-term effects observed during thyroid hormone disturbances, the hemodynamic actions explain why the cardiovascular manifestations are emblematical and sensitive to thyroid dysfunction. However, when trying to integrate pragmatically these data, one should be aware of the risk of thinking simplistically. For example, thyroid hormones stimulate catecholamine synthesis, RAA system and erythropoietin synthesis, all in an adaptive manner to improve hemodynamics and metabolism, but long term this "adaptive" mechanism becomes maladaptive and leads to cardiac dysfunction; adding thyroid hormone inhibitors to conventional medication in this setting seems a rational approach. On the other hand, decreased level of thyroid hormones, even at the subclinical level of thyroid disease, has deleterious cardiovascular effects [7] increasing resistance, favoring cardiac remodeling and impairing cardiac function; therefore, in this case, adding thyroid hormone replacement could improve the condition. Hence the thyroid hormones' physiological effects on cardiovascular system are not a simple "on-off" mechanism, but the result of a very fine and delicate tuning.

The direct effect exerted by $\mathrm{T}_{3}$ on the cardiac adaptive machinery is even more complicated, more subtle and less comprehensible. This special mini review in Heart Failure Reviews deals with some of these aspects in expert hands. Thyroid hormones are legitimate players in the complicated mechanisms of cardioprotection as they influence important cell signaling molecules, are regulators of mitochondrial structure and function and are implicated in the regenerative process of myocardium and affect almost all cardiac structures (myocyte, interstitial cells and microvascular cells). $\mathrm{T}_{3}$ modulates directly or via microRNA small molecules the mitochondrial function, biogenesis and quality through multiple regulation loops including reperfusion injury signaling kinase (RISK) and tumor suppressor protein $\mathrm{p} 53 . \mathrm{T}_{3}$ prevents the hypoxia mediated increase in p53 which occurs after myocardial infarction and promotes apoptosis, mitochondrial dysfunction and progression to heart failure [8]. The clinical relevance of thyroid hormones protection is currently tested in the "Acute and long-term effects of thyroid hormone replacement therapy in patients with ST-Elevation Myocardial Infarction (STEMI) and borderline/reduced triiodothyronine levels" (THiRST) study. Very recently Lerman et al. reported in a cohort of 2430 who underwent percutaneous coronary intervention a significant increase in major cardiovascular events in patients with low level of thyroid hormones and overt or subclinical hypothyroidism [9]. Increasing evidence also supports the role of thyroid hormones in preventing deleterious cardiac remodeling. Many interrelated mechanisms could account for these complex actions including antiapoptotic effects, decrease of collagen expression, increase in contractility [10], switch from maladaptive to adaptive hypertrophy [11], improving mitochondrial metabolism and promoting angiogenesis and cell viability. Interesting, the anti-remodeling thyroid hormone activity is not limited to ventricle, but also prevents atrial fibrosis and atrial fibrillation induction [12].

In chronic heart disease (heart failure, coronary heart disease), the systemic pro-inflammatory response associates parallel increase in interleukin 6 and tumor necrosis alpha with decrease in $\mathrm{T}_{3}$; the last is mediated through the blockade of iodothyronine deiodinase synthesis and activity [2]. The decrease in $\mathrm{T}_{3}$ concentration is proportional to the heart failure severity. The same kind of alteration in gene expression is observed both in primary hypothyroidism and in secondary low $T_{3}$ syndrome accompanying heart failure [13]. This pathological state perpetuates the deleterious cardiac remodeling and contributes to heart failure progression. In euthyroid patients with coronary artery disease, a similar pattern of low $\mathrm{T}_{3}$ was observed and correlates with outcome [14].

The short presentation of the exciting story which follows in this issue of the Journal would be incomplete without remembering that endogenous rapid acting derivative of thyroid hormones, as 3-Iodothyronamine $\left(T_{1} A M\right)$ also acts as a multi-target ligand and exerts important cardiac, cardioprotective and metabolic actions not yet fully understood, diversifying even more the spectrum of interference of thyroid hormones with the cardiovascular system. 


\section{Compliance with ethical standards}

Conflict of interest No conflict of interest to declare for this paper.

\section{References}

1. Sussman MA (2001) When the thyroid speaks the heart listens. Circ Res 89:557-559

2. Danzi S, Klein I (2014) Thyroid disease and the cardiovascular system. Endocrinol Metab Clin North Am 43:517-528

3. Yu J, Koenig RJ (2006) Induction of type 1 iodothyronine deiodinase to prevent the nonthyroidal illness syndrome in mice. Endocrinology 147:3580-3585

4. Bassett JH, Harvey CB, Williams GR (2003) Mechanisms of thyroid hormone receptor-specific nuclear and extra nuclear actions. Mol Cell Endocrinol 31(213):1-11

5. Visser WE, Friesema EC, Visser TJ (2011) Minireview: thyroid hormone transporters: the knowns and the unknowns. Mol Endocrinol 25:1-14

6. Klein I (2015) Endocrine disorders and cardiovascular disease. In: Mann MDL, Zipes DP, Libby P, Bonow RO (eds) Braunwald's heart disease: a textbook of cardiovascular medicine, 10th edn. Saunders, pp 1798-1799

7. Gencer B, Collet TH, Virgini V, Auer R, Rodondi N (2013) Subclinical thyroid dysfunction and cardiovascular outcomes among prospective cohort studies. Endocr Metab Immune Disord Drug Targets 13:4-12

8. Forini F, Kusmic C, Nicolini G, Mariani L, Zucchi R, Matteucci $M$ et al (2014) Triiodothyronine prevents cardiac ischemia/ reperfusion mitochondrial impairment and cell loss by regulating $\mathrm{miR} 30 \mathrm{a} / \mathrm{p} 53$ axis. Endocrinology 155:4581-4590

9. Zhang M, Sara JD, Matsuzawa Y, Gharib H, Bell MR, Gulati R, et al (2016) Clinical outcomes of patients with hypothyroidism undergoing percutaneous coronary intervention. Eur Heart J. doi:10.1093/eurheartj/ehv737

10. Cokkinos DV, Pantos C (2009) Thyroid hormones and their action on the myocardium. Bull Acad Natl Med. 193(2):327-336 (discussion 336-8)

11. Cheng SY, Leonard JL, Davis PJ (2010) Molecular aspects of thyroid hormone actions. Endocr Rev 31:139-170

12. Zhang Y, Dedkov EI, Lee B, Li Y, Pun K, Gerdes AM (2014) Thyroid hormone replacement therapy attenuates atrial remodeling and reduces atrial fibrillation inducibility in a rat myocardial infarction-heart failure model. J Card Fail 20(12):1012-1019

13. Danzi S, Klein I (2012) Thyroid hormone and the cardiovascular system. Med Clin North Am 96:257-268

14. Coceani M, Iervasi G, Pingitore A, Carpeggiani C, L'Abbate A (2009) Thyroid hormone and coronary artery disease: from clinical correlations to prognostic implications. Clin Cardiol $32: 380-385$ 